SCIENCE CHINA

Physics, Mechanics \& Astronomy

\title{
Erratum to: Influence of the time-step on the production of free nucleons and pions from heavy-ion collisions around $1 \mathrm{GeV} /$ nucleon [Sci. China-Phys. Mech. Astron. 59, 122011 (2016)]
}

\author{
LiYan Zou ${ }^{1,2}$, Miao Li ${ }^{1,2}$, ChenChen Guo ${ }^{3}$, YongJia Wang ${ }^{1 *}$, QingFeng $\mathrm{Li}^{1,4^{*}}$, and Ling Liu ${ }^{2}$ \\ ${ }^{1}$ School of Science, Huzhou University, Huzhou 313000, China; \\ ${ }^{2}$ College of Physics Science and Technology, Shenyang Normal University, Shenyang 110034, China; \\ ${ }^{3}$ Shanghai Institute of Applied Physics, Chinese Academy of Sciences, Shanghai 201800, China; \\ ${ }^{4}$ Institute of Modern Physics, Chinese Academy of Sciences, Lanzhou 730000, China
}

Received November 1, 2016; accepted November 2, 2016; published online November 22, 2016
Citation: L. Y. Zou, M. Li, C. C. Guo, Y. J. Wang, Q. F. Li, and L. Liu, Erratum to: Influence of the time-step on the production of free nucleons and pions from heavy-ion collisions around $1 \mathrm{GeV} /$ nucleon [Sci. China-Phys. Mech. Astron. 59, 122011 (2016)], Sci. China-Phys. Mech. Astron. 60, 022051 (2017), doi: $10.1007 / \mathrm{s} 11433-016-0432-4$

In the References of the original publication [1] of this paper, the authors of ref. [26] should be "S. A. Bass, et al. (UrQMD Collaboration)", the authors of ref. [27] should be "M. Bleicher, et al. (UrQMD Collaboration)". The authors of ref. [35] should be "W. Reisdorf, et al. (FOPI Collaboration)", the authors of ref. [38] should be "W. Reisdorf, et al. (FOPI Collaboration)".

1 L. Y. Zou, M. Li, C. C. Guo, Y. J. Wang, Q. F. Li, and L. Liu, Sci. China-Phys. Mech. Astron. 59, 122011 (2016).

*Corresponding authors (YongJia Wang, email: wangyongjia@zjhu.edu.cn;

QingFeng Li, email: liqf@zjhu.edu.cn) 